ARTICULOS ORIGINALES

Rev Chil Salud Pública 2020,

Vol 24(1): $40-48$

\title{
FACTORES SOCIODEMOGRÁFICOS Y CLÍNICOS ASOCIADOS A LA SATISFACCIÓN USUARIA DE CUIDADORES EN UN HOSPITAL PEDIÁTRICO DE ALTA COMPLEJIDAD.
}

\author{
SOCIODEMOGRAPHIC AND CLINICAL FACTORS ASSOCIATED WITH \\ CAREGIVERS' SATISFACTION IN A TERTIARY PEDIATRIC HOSPITAL.
}

\section{RESUMEN}

Introducción: La calidad y seguridad de la atención constituyen pilares fundamentales del sistema de salud, basándose en el respeto por la dignidad y autonomía de los usuarios. Esto demanda que la atención considere características propias de las personas al evaluar su satisfacción con sus servicios; sin embargo, las encuestas de satisfacción usuaria suelen indagar solamente en generalidades como infraestructura y tiempos de atención. El objetivo de este trabajo era evaluar los factores socio-demográficos y clínicos asociados a la satisfacción usuaria de padres/cuidadores en un hospital pediátrico de alta complejidad en Santiago, Chile.

Materiales y Métodos: Se aplicó la encuesta Pediatric Acute Care, adaptada al castellano, a 304 padres/cuidadores de pacientes pediátricos. Se analizó el comportamiento psicométrico del instrumento, y se aplicó un modelo lineal generalizado para evaluar las características socio-demográficas y clínicas asociadas al puntaje de satisfacción usuaria.

Resultados: Los padres/cuidadores que perciben la salud del niño/a como "buena" presentan, en promedio, 4,64 puntos más de satisfacción en comparación a quienes consideran la salud como "mala" (IC95\% 0,9 - 8,38; p=0,015). Del mismo modo, a medida que aumenta la edad del niño/a en un mes, la satisfacción aumenta en 0,026 puntos (IC95\% 0,01 - 0,041; $p=0,001)$. No obstante, la satisfacción disminuye en 0,06 puntos por cada día que el niño/a pasa hospitalizado/a (IC95\% -0,69--0,003; $p=0,034$ ), ajustando por otras variables.

Discusión: La satisfacción usuaria de padres/cuidadores de niños/as hospitalizados está asociada a características personales de los mismos. Los profesionales de la salud deben considerar estas características con el objetivo de otorgar una atención de calidad, ajustada a las necesidades de los usuarios.

Palabras clave: Satisfacción del Paciente; Hospitales Pediátricos; Determinantes Sociales de la Salud; Prestación de Atención de Salud

\section{ABSTRACT}

Carolina Concha-Gutiérrez. Escuela de Enfermería, Universidad de Los Andes, Santiago, Chile.

Edith Rivas-Riveros. Escuela de Enfermería, Universidad de La Frontera, Temuco, Chile.

Pilar Espinoza-Quiroz. Dirección de Postgrado, Investigación y Vinculación Internacional, Universidad San Sebastián, Santiago, Chile.

Yasna K Palmeiro-Silva. Institute for Global Health, University College London, Londres, Reino Unido. Escuela de Enfermería, Universidad de Los Andes, Santiago, Chile. ykpalmei@uc.cl

Recibido el 01-04-2019 Aceptado el 20-10-2019
Introduction: Assuring the quality of care and patient safety are central pillars of health systems, in order to respect patients' dignity and autonomy. On the basis of these principles, healthcare systems should consider users' personal characteristics to evaluate their satisfaction with services; however, most user satisfaction surveys only ask about general issues, such as infrastructure and wait times. The objective of this study was to evaluate socio-demographic and clinical factors related to parent/caregiver's user satisfaction in a tertiary pediatric hospital located in Santiago, Chile.

Materials and Methods: The Pediatric Acute Care questionnaire was adapted and applied to 304 parents/caregivers. Psychometric characteristics were evaluated, and a generalized linear model was applied to obtain socio-demographic and clinical factors related to the user satisfaction score.

Results: Parents/caregivers who perceive their child's health as 'good' obtained, on average, 4.64 more satisfaction points than those who consider their child's health as 'bad' (IC95\% $0.9-8.38 ; p=0.015)$. Likewise, as a child's age increases by one month, satisfaction increases by 0.0026 points (IC95\% $0.01-0.041 ; p=0.001$ ). Nonetheless, each additional day a child is hospitalized decreases user satisfaction by 0.06 points (IC95\% $-0.69--0.003 ; p=0.034$ ), holding other variables constant.

Discussion: User satisfaction is associated with parents/caregivers' personal characteristics. Health workers should consider these characteristics in order to deliver better care, adjusted to their users' needs.

Key Words: Patient Satisfaction; Hospitals, Pediatric; Social Determinants of Health; Delivery of Health Care 


\section{INTRODUCCIÓN}

La calidad y seguridad asistencial se han transformado en pilares fundamentales en la actual gestión de salud, obligando a instituciones de salud a establecer diversos estándares en esos ámbitos.

En 1990, el Instituto de Medicina de Estados Unidos definió la calidad en salud como "el grado en que los servicios de salud incrementan la probabilidad de resultados deseados para los individuos y la población y son consistentes con el conocimiento profesional actual" ${ }^{\prime 1}$. Junto con ello, Donabenian sugirió que la calidad en salud se debe evaluar en términos de estructura, procesos y resultados ${ }^{2}$, y define siete atributos de la calidad en salud: eficacia, efectividad, eficiencia, optimalidad, aceptabilidad, legitimidad y equidad, concluyendo que una atención en salud de calidad debe considerar las preferencias y características sociales de los pacientes ${ }^{3}$.

En este sentido, el sistema de salud chileno ha presentado importantes avances para resguardar una atención de calidad. Ejemplo de ello son la Ley $\mathrm{N}^{\circ} 19.966$, donde se define la garantía de calidad, y la Ley $N^{\circ} 20.584$, que regula los derechos y deberes que tienen las personas en relación con acciones vinculadas a su atención en salud, poniendo a la persona como centro de la ley, respetando su dignidad, autonomía e igualdad.

Consecuentemente, al poner a la persona como centro del sistema de salud, es lógico pensar que los indicadores de funcionamiento se centren en los usuarios, de esta manera, la evaluación de la percepción o satisfacción usuaria toma especial relevancia.

$\mathrm{Al}$ analizar la satisfacción usuaria en Chile, aparecen múltiples encuestas realizadas por el Ministerio de Salud ${ }^{4,5}$, la Superintendencia de Salud ${ }^{6}$, y distintos centros de salud ${ }^{7}$. Desafortunadamente, estas mediciones no han sido realizadas en base preguntas/respuestas comparables entre las diferentes encuestas. Además, la mayoría de las preguntas son del ámbito de infraestructura, tiempos de atención, costos y cobertura, aspectos que evalúan de manera global a la población, dejando de lado preferencias o características personales, como por ejemplo edad, nivel educacional, experiencias previas con el sistema de salud, etc., aspectos que son fundamentales al momento de otorgar una atención de salud equitativa.

De especial importancia son los niños/as usuarios de un sistema de salud, ya que en pocas ocasiones se les considera como usuarios "activos" del sistema y son los padres/cuidadores quienes toman ese rol. Lamentablemente, escasa evidencia en español muestra esta perspectiva; adicionalmente, no existe un instrumento en Chile que evalúe la satisfacción usuaria de padres/cuidadores de pacientes pediátricos.

Con el objetivo de aportar en este ámbito, este estudio evalúa los factores socio-demográficos y clínicos que se asocian a la satisfacción usuaria de cuidadores en un hospital pediátrico de alta complejidad en la ciudad de Santiago, Chile.

\section{MATERIAL Y MÉTODO}

Diseño del estudio y población: Se realizó un estudio transversal que consideró a padres/cuidadores de pacientes pediátricos de un hospital de alta complejidad en la ciudad de Santiago, Chile. Fueron incluidos en el estudio a padres/cuidadores cuyos hijos/as o parientes presentaran hasta 19 años de edad y patologías de resolución quirúrgica con estadía hospitalaria mayor a 48 horas. El tamaño muestral calculado fue de 307 sujetos y el muestreo fue no probabilístico.

Recolección de datos: Los datos fueron recolectados por seis meses hasta abril 2016. Durante la hospitalización del paciente, un investigador (CCG) aplicó una encuesta a padres/cuidadores que indagó lo siguiente:

- Información socio-demográfica del padre/cuidador. Incluyó edad, sexo, nacionalidad, estado civil, nivel educacional, tipo de trabajo (en el hogar/fuera del hogar), rol en la familia del paciente, residencia principal y percepción de la salud del paciente.

- Información socio-demográfica y clínica del paciente. Incluyó edad, sexo, servicio de hospitalización (cirugía infantil, lactante/segunda infancia, neonatología y unidad de paciente crítico pediátrica - UCIP), número de hospitalizaciones en el último año y días de hospitalización actual.

- Satisfacción usuaria del padre/cuidador. Se evaluó a través de una encuesta basada en los instrumentos Pediatric Inpatient Survey ${ }^{8}$ y Pediatric Acute Care (PCA). Esta última consta de 37 preguntas, agrupadas en ocho indicadores: impresión general; acceso a cuidados y servicios; consideración y respeto; continuidad y transición; coordinación e integración del cuidado; información, comunicación y educación; relación entre padres y equi- 
po de salud; y confort físico ${ }^{9}$. La encuesta PCA fue validada al castellano por Eulmesekian et al., mostrando una consistencia interna de 0.8710 .

Se comparó la encuesta PCA y la encuesta en castellano. Se analizaron las diferencias semánticas y se solicitó autorización para el uso de la encuesta. Se realizó una validez de fachada que resultó en una adaptación semántica e incorporación de dos preguntas (iel personal de salud que atiende a su hijo porta credencial de identificación?, ¿la sala donde se encuentra su hijo está limpia?). Posteriormente, se realizó una evaluación piloto con una muestra de 5 padres/cuidadores del mismo hospital en estudio. El cuestionario aplicado se encuentra en anexos.

El protocolo del estudio fue aprobado por el Comité de Ética del Servicio de Salud Metropolitano Oriente. Además, todos los sujetos otorgaron su consentimiento por escrito previo a los procedimientos del estudio.

Análisis estadístico: La muestra se analizó descriptivamente, luego se evaluó la consistencia interna para el cuestionario considerando las preguntas de la encuesta validada por Eulmesekian et al. con el objetivo de comparar ambos estudios.

El puntaje de satisfacción para cada indicador se obtuvo calculando la media de puntajes obtenidos de las preguntas pertenecientes al indicador. Por otro lado, el puntaje de satisfacción general se obtuvo a través de la media de los puntajes de satisfacción de cada indicador ${ }^{9,10}$.

Se compararon los puntajes de satisfacción por indicador y general, considerando variables socio-demográficas y clínicas. Dada la distribución de las variables, se aplicaron los test de U-Mann-Whitney o Kruskal-Wallis según fue apropiado, además, para variables cuantitativas se evaluó la correlación de Spearman. Para variables categóricas se consideró el uso del test Chi2 o exacto de Fisher.

Para evaluar los factores socio-demográficos relacionados a la satisfacción usuaria global, se analizó un modelo lineal generalizado, considerando una distribución Gaussiana y función identity. En un primer modelo, se incorporaron todos los factores socio-demográficos, posteriormente se fueron descartando, hasta la obtención de un modelo con menor criterio de Akaike.

Los análisis se realizaron utilizando el programa estadístico STATA 15.1 considerando un nivel de significancia de $\mathrm{p}<0.05$.

\section{RESULTADOS}

La muestra estuvo compuesta por 304 padres/cuidadores, quienes respondieron completamente el cuestionario. La tabla 1 muestra las características

Tabla 1. Características socio-demográficas de padres/cuidadores.

\begin{tabular}{|c|c|}
\hline \multicolumn{2}{|l|}{ Variable } \\
\hline Edad en años, me(p25-p75) ${ }^{a}$ & $35(28-42)$ \\
\hline \multicolumn{2}{|l|}{ Sexo } \\
\hline Hombre, n (\%) & $46(15.13)$ \\
\hline Mujer, n (\%) & $258(85.87)$ \\
\hline \multicolumn{2}{|l|}{ Nacionalidad } \\
\hline Chileno, n (\%) & $286(94.08)$ \\
\hline Extranjero, n (\%) & $18(5.92)$ \\
\hline \multicolumn{2}{|l|}{ Estado Civil ${ }^{b}$} \\
\hline En pareja, n (\%) & $95(31.25)$ \\
\hline Sin pareja, n (\%) & $209(68.75)$ \\
\hline \multicolumn{2}{|l|}{ Nivel Educacional } \\
\hline Ed. Básica, n (\%) & $48(15.79)$ \\
\hline Ed. Media, n (\%) & $156(51.32)$ \\
\hline Ed. Superior, n (\%) & $100(32.89)$ \\
\hline \multicolumn{2}{|l|}{ Tipo de trabajo } \\
\hline En el hogar, $n(\%)$ & $281(92.43)$ \\
\hline Fuera del hogar, n (\%) & $23(7.57)$ \\
\hline \multicolumn{2}{|l|}{ Rol en la familia del paciente } \\
\hline Padre, n (\%) & $38(12.5)$ \\
\hline Madre, n (\%) & $223(73.36)$ \\
\hline Otro, n (\%) & $43(14.14)$ \\
\hline \multicolumn{2}{|l|}{ Residencia principal } \\
\hline Región Metropolitana, n (\%) & $191(62.83)$ \\
\hline Otra región, n (\%) & $113(37.17)$ \\
\hline \multicolumn{2}{|c|}{ Percepción del cuidador sobre la salud del paciente } \\
\hline Mala, n (\%) & $22(7.24)$ \\
\hline Aceptable, n (\%) & $105(34.54)$ \\
\hline Buena, n (\%) & $117(58.22)$ \\
\hline
\end{tabular}


Tabla 2. Características socio-demográficas y clínicas de pacientes pediátricos.

\begin{tabular}{lll}
\hline Variable & & \\
\hline Servicio de hospitalización & 153 & $(50.33)$ \\
Cirugía infantil, $\mathrm{n}(\%)$ & 69 & $(22.7)$ \\
Segunda Infancia, $\mathrm{n}(\%)$ & 69 & $(22.7)$ \\
Lactante, $\mathrm{n}(\%)$ & 13 & $(4.28)$ \\
UCIP $\mathrm{a}, \mathrm{n}(\%)$ & 54.5 & $(15-132)$ \\
Edad en meses, me(p25-p75) b & & $(48.68)$ \\
Sexo & 148 & $(51.32)$ \\
Hombre, $\mathrm{n}(\%)$ & 156 & \\
Mujer, $\mathrm{n}(\%)$ & & $(53.49)$ \\
Número de hospitalizaciones en el último año & 193 & $(30.26)$ \\
Una (incluye actual), $\mathrm{n}(\%)$ & 92 & $(6.25)$ \\
Entre 2 a 5 veces, $\mathrm{n}(\%)$ & 19 & $(3-9)$ \\
Más de 5 veces, $\mathrm{n}(\%)$ & 5 & \\
Días de hospitalización actual, me(p25-p75) & & \\
\hline
\end{tabular}

a UCIP: Unidad de Cuidados Intensivos Pediátrica

b me(p25-p75): mediana (percentil 25 y percentil 75)

socio-demográficas de padres/cuidadores y la tabla 2 muestra las características socio-demográficas y clínicas de los pacientes pediátricos.

El cuestionario aplicado presentó una consistencia interna global de 0.83 (con 38 ítems totales). En la tabla 3 se observan las consistencias internas para cada indicador.

Tabla 3. Consistencias internas de cada indicador.

\begin{tabular}{lc}
\hline Indicador & Consistencia interna \\
\hline Impresión General & 0.53 \\
Acceso a cuidados y servicios & 0.70 \\
Consideración y respeto & 0.68 \\
Coordinación e integración del cuidado & 0.30 \\
Información, comunicación y educación & 0.46 \\
Relación entre padres y equipo de salud & 0.63 \\
Confort físico & 0.62 \\
Continuidad y transición del cuidado & 0.72 \\
\hline
\end{tabular}


Se observa que el servicio de UCIP presenta mejores niveles de satisfacción en la mayoría de los indicadores, excepto en relación entre padres y equipo de salud, confort físico y continuidad del cuidado (tabla 4). La satisfacción usuaria global fue estadísti- camente diferente entre los servicios, observándose que el servicio de cirugía presentó el mayor puntaje de satisfacción usuaria global, seguido por la UCIP $(p=0.009)$.

Tabla 4. Medianas de puntajes de satisfacción usuaria por indicador y global, según servicio de hospitalización.

\begin{tabular}{|c|c|c|c|c|c|}
\hline Indicador, me(p25-p75) & $\begin{array}{l}\text { Cirugía } \\
(n=153)\end{array}$ & $\begin{array}{c}\text { S. Infancia } \\
(n=69)\end{array}$ & $\begin{array}{l}\text { Lactante } \\
(n=69)\end{array}$ & $\begin{array}{l}\text { UCIP } \\
(n=13)\end{array}$ & p-value \\
\hline Impresión General & $87.5(81.25-93.75)$ & $87.5(75-93.75)$ & $87.5(81.25-93.75)$ & 87.5 (81.25-93.75) & 0.2981 \\
\hline $\begin{array}{l}\text { Acceso a cuidados y } \\
\text { servicios }\end{array}$ & $92.86(85.71-96.43)$ & $92.86(85.71-96.43)$ & $92.86(85.71-96.43)$ & $96.43(89.29-100)$ & 0.5305 \\
\hline Consideración y respeto & $90(85-100)$ & $90(85-95)$ & $90(85-100)$ & $95(85-100)$ & 0.6250 \\
\hline $\begin{array}{l}\text { Coordinación e integración } \\
\text { del cuidado }\end{array}$ & 93.75 (75-93.75) & 93.75 (81.25-93.75) & $87.5(75-100)$ & $100(75-100)$ & 0.5100 \\
\hline $\begin{array}{l}\text { Información, comunicación } \\
\text { y educación }\end{array}$ & $100(80-100)$ & $87.5(70-100)$ & $87.5(75-100)$ & $100(80-100)$ & 0.0753 \\
\hline $\begin{array}{l}\text { Relación entre padres y } \\
\text { equipo de salud }\end{array}$ & $100(80-100)$ & $90(80-100)$ & $90(80-100)$ & $90(80-100)$ & 0.3916 \\
\hline Confort físico & $100(87.5-100)$ & $100(75-100)$ & $87.5(75-100)$ & $87.5(75-100)$ & 0.0001 \\
\hline $\begin{array}{l}\text { Continuidad y transición } \\
\text { del cuidado }\end{array}$ & $20(20-20)$ & $20(20-20)$ & $20(20-20)$ & $20(10-20)$ & 0.0879 \\
\hline Satisfacción Usuaria Global & $82.05(77.92-85.02)$ & $79.62(71.61-84.53)$ & $78.15(71.45-82.57)$ & 78.84 (75.2-85.94) & 0.0090 \\
\hline
\end{tabular}

${ }^{a}$ me(p25-p75): mediana (percentil 25 y percentil 75)

En relación al porte de la credencial de identificación, la mayor parte de los padres identifica que el personal siempre porta la credencial, pero esta proporción varía respecto al servicio $(\mathrm{p}=0.001)$. Por otra parte, no existió diferencia en la opinión de los padres/cuidadores respecto a la limpieza de la sala y el servicio $(p=0.376)$.

Al analizar los puntajes según el sexo de los padres/cuidadores, las mujeres presentan menor satisfacción en los indicadores de relación entre padres y equipo de salud $(p=0.0379)$ y confort físico $(p=0.0151)$, sin embargo, los demás puntajes fueron similares.

Por otro lado, los padres/cuidadores extranjeros, presentaron menores puntajes de satisfacción en los indicadores de acceso a cuidados y servicios $(p=0.0069)$, confort físico $(p=0.0033)$ y satisfacción global $(p=0.0016)$.

Según el rol que cumple la persona encuestada, se observó que las madres, presentan menor puntaje de satisfacción en los indicadores de acceso a cuidados y servicio $(p=0.0424)$, relación entre padres y equipo de salud $(p=0.0068)$, y satisfacción general $(\mathrm{p}=0.0347)$.

Los padres/cuidadores que perciben la salud del paciente como "mala", presentan menores puntajes de satisfacción en los indicadores de impresión general $(p=0.0430)$, acceso a cuidados y servicios $(p=0.0037)$, confort físico $(p=0.0218)$ y satisfacción general $(p=0.0017)$.

La satisfacción en los indicadores de impresión general y continuidad y transición del cuidado es menor en el grupo de padres/cuidadores que han tenido a su hijo/a hospitalizado más de 5 veces en el año $(p=0.0063$ y $p=0.0187$, respectivamente)

Los puntajes de satisfacción para cada indicador y satisfacción global no mostraron diferencias significativas al evaluar según residencia, tipo de trabajo, nivel educacional, estado civil y el sexo del paciente. 
Adicionalmente, por cada año más que tengan los padres/cuidadores, el puntaje de satisfacción en confort físico aumenta en 0.17 puntos $(p=0.0039)$ y la satisfacción global en 0.22 puntos $(\mathrm{p}=0.0002)$. De manera similar, la edad del paciente se correlacionó positivamente con los indicadores de coordinación e integración del cuidado ( $\mathrm{rho}=0.12, \mathrm{p}=0.0421$ ); relación entre padres y equipo de salud ( $\mathrm{rho}=0.13$, $\mathrm{p}=0.019$ ); confort físico ( $\mathrm{rho}=0.2944, \mathrm{p}=0.0001$ ), continuidad y transición del cuidado (rho $=0.12$, $\mathrm{p}=0.0389) ; \quad$ y satisfacción global (rho=0.22, $\mathrm{p}=0.0001$ ).

Derivado del modelo de regresión, se obtiene que las variables de percepción de salud de los padres/ cuidadores, la edad del niño/a y el número de días de hospitalización actual son significativas en la explicación de la satisfacción usuaria global, no así la residencia, el sexo de los padres/cuidadores y el rol de los mismos. Se estimó que los padres/cuidadores que perciben la salud de su hijo/a como buena, presentan en promedio, 4.64 puntos más de satisfacción que quienes la consideran mala (IC95\% 0.9-8.38; $\mathrm{p}=0.015)$. Por otra parte, por cada mes de incremento en la edad del niño/a hospitalizado/a, la satisfac- ción usuaria de los padres/cuidadores aumenta en 0.026 puntos (IC95\% 0.01-0.041; p=0.001) y por cada día que el niño/a pasa hospitalizado/a, la satisfacción usuaria de los padres/cuidadores disminuye en 0.06 puntos (IC95\% $-0.69--0.0028 ; p=0.034$ ), ajustando por las demás variables (Tabla 5).

\section{DISCUSIÓN}

Este estudio demuestra que la satisfacción usuaria de los padres/cuidadores está asociada a los factores de percepción de salud del paciente pediátrico por parte de los padres/cuidadores, edad del niño/a hospitalizado/a y el número de días de hospitalización. Desde un punto de vista técnico, el cuestionario aplicado muestra una consistencia interna de 0.83, considerada buena, además de ser similar a la hallada por Eulmesekian et al. ${ }^{10}$.

La muestra estudiada refleja "el cuidado habitual" de los niños/as en Chile y Latinoamérica. La mayoría de los encuestados son mujeres y madres de los niños/as hospitalizados, lo que concuerda que en la mayoría de los casos, el cuidado recae en la madre o en alguna figura materna ${ }^{11}$. Este fenómeno coincide

Tabla 5. Resultados del modelo de regresión para satisfacción usuaria global.

\begin{tabular}{|c|c|c|c|c|c|}
\hline & Coef $^{\mathrm{a}}$ & Error Est $^{b}$ & P-valor & \multicolumn{2}{|c|}{ Intervalo de Confianza 95\% } \\
\hline Región & 1.8149 & 1.0488 & 0.084 & -0.2407 & 3.8706 \\
\hline \multicolumn{6}{|l|}{ Sexo } \\
\hline Mujer & 0.1779 & 2.9850 & 0.952 & -5.6725 & 6.0284 \\
\hline \multicolumn{6}{|l|}{ Rol } \\
\hline Madre & 6.0284 & 3.2583 & 0.286 & -9.8622 & 2.9103 \\
\hline Otro & 0.2236 & 3.3953 & 0.947 & -6.4310 & 6.8784 \\
\hline \multicolumn{6}{|l|}{ Percepción Salud } \\
\hline Aceptable & 2.2510 & 1.9527 & 0.249 & -1.5762 & 6.0782 \\
\hline Buena & 4.6417 & 1.9082 & 0.015 & 0.9015 & 8.3818 \\
\hline Edad del niño & 0.0257 & 0.0077 & 0.001 & 0.0105 & 0.0410 \\
\hline Días hospitalización actual & -0.0359 & 0.0169 & 0.034 & -0.0690 & -0.0027 \\
\hline Constante & 75.332 & 2.3050 & 0.000 & 70.8144 & 79.8501 \\
\hline
\end{tabular}

\footnotetext{
${ }^{\text {a }}$ Coef: Coeficiente estimado

b Error Est: Error Estándar.

$\mathrm{AIC}=7.09$
} 
con que las personas con el rol de madre, tienen menor puntaje en los indicadores de acceso a cuidados y servicios, relación entre padres y equipo de salud y satisfacción general, ya que estos indicadores se relacionan al manejo de información de los cuidados y el involucramiento con los mismos.

El hecho que los padres/cuidadores que perciban la salud del paciente como "mala" se relacione a una menor satisfacción usuaria global, puede ser explicado por las experiencias previas de la persona. En otras palabras, si el padre/cuidador considera que la salud del hijo/a es "mala", lo más probable que las experiencias pasadas, que se relacionen a la salud del hijo/a, no sean positivas. Así, experiencias previas condicionan la experiencia actual. Este haIlazgo es similar con lo reportado por Paul et al. y Xiao et al., donde pacientes con peor salud percibida, presentaron peor satisfacción usuaria con el sistema de salud ${ }^{12,13}$. En esta línea, es fundamental que los profesionales de la salud consideren este punto al momento de interactuar con padres/cuidadores, teniendo en cuenta que las experiencias y conocimientos previos marcan la vida de las personas, ya sea positiva o negativamente $\mathrm{e}^{14-17}$.

Por otra parte, la satisfacción usuaria global es mayor a medida que el hijo tiene mayor edad. Esto podría explicarse debido a la percepción de vulnerabilidad y dependencia de los hijos más pequeños por parte de los padres, lo que se refleja en el nivel de satisfacción. Sin embargo, Moreno-Monsiváis et al., observó que la edad del hijo y la percepción de su estado de salud no fueron significativos para la satisfacción de las madres ${ }^{18}$. Esta discrepancia podría ser debido a que los instrumentos fueron diferentes y en ese estudio los pacientes presentaban patologías crónicas.

Adicionalmente, al aumentar el número de días de la hospitalización actual, la satisfacción usuaria disminuye, lo que se soporta en la idea de que la hospitalización no es una experiencia placentera, es agotadora y disruptiva en términos de la dinámica familiar y social. En este sentido, a mayor estadía hospitalaria, los padres/cuidadores sienten alejamiento "obligado" con sus hijos/as, lo que aumentaría el agotamiento físico y mental, como lo plantea Franck et al. ${ }^{19}$ y Stremler et al. ${ }^{20}$. Este distress recaería en la satisfacción usuaria de los padres, por lo que el sistema de salud debiera considerar que la hospitalización prolongada de un niño va a provocar una serie de efectos psicológicos y emocionales en la familia, los que debe ser considerado en la pla- nificación del cuidado y la comunicación con los padres/cuidadores.

En general, el efecto de las variables asociadas a la satisfacción usuaria de padres/cuidadores en este estudio, podría ser manejado a través de la respuesta de parte de los profesionales de la salud y las instituciones, ya que los padres/cuidadores de niños/ as hospitalizados/as son vulnerables y pueden ser afectados tanto psíquica como emocionalmente por estresores del medioambiente, el cual considera la interacción con el personal de salud. Diferentes autores plantean que el soporte centrado en la familia y sus necesidades es fundamental en ellos, ya que por una parte disminuye el estrés percibido, mejora la comunicación entre la familia y el personal de salud, como también mejora la satisfacción usuaria de la atención de salud ${ }^{21-24}$.

Los profesionales de la salud deben considerar características socio-demográficas específicas al momento de comunicarse con los padres/cuidadores, ya que estas juegan un rol modificador entre la atención en salud y la satisfacción usuaria ${ }^{25}$. Por ejemplo, el idioma o ciertas creencias culturales en el caso de padres/cuidadores extranjeros o de diferente etnia ${ }^{26}$. Además, se deben considerar los miedos, cuestionamientos, dudas y experiencias pasadas de los padres/cuidadores. Esto le permite al profesional de la salud adecuar el mensaje que se desea transmitir, así la comunicación será efectiva y empática ${ }^{27-30}$.

Finalmente, la medición de la satisfacción usuaria presenta aspectos positivos tanto para el sistema de salud y los usuarios. De acuerdo a Ilioudi et al., la evaluación de la satisfacción usuaria debiera impactar la gestión de las instituciones y la atención en salud, lo que se describe "bottom up" ${ }^{31}$, guiando los procesos de mejora dentro de una institución de salud ${ }^{32}$. Adicionalmente, pacientes satisfechos podrían presentar mejor adherencia al tratamiento y mejores outcomes, por lo que se debe considerar la importancia de las "buenas experiencias", más allá que la satisfacción usuaria y la resolución del problema de salud, ya que las experiencias marcan el comportamiento y confianza posterior de las personas sobre el sistema, instituciones y equipo de sa$\operatorname{lud}^{33,34}$.

Como limitación del estudio, una muestra intencionada de una institución de salud, limita la extrapolación de resultados. Además, al ser un estudio transversal, no se puede inferir si la satisfacción usuaria varia a lo largo de la hospitalización del niño/a y/o si existen variables que cambian a lo largo 
del tiempo. Finalmente, sería de mucho interés el desarrollo o validación de un instrumento que permita evaluar la satisfacción usuaria o experiencia con la atención en salud desde el punto de vista de los niños/as, ya que así se los valida como personas con derechos y experiencias propias, respetando su dignidad y autonomía.

\section{Financiamiento:}

Este estudio no contó con fuentes de financiamiento externas.

Declaración de Intereses: Los autores declaran no tener conflicto de interés de ningún tipo.

\section{Agradecimientos:}

Los autores agradecen a los padres y cuidadores que participaron en este estudio, y a los médicos y enfermeras que facilitaron el trabajo de campo. YKPS agradece al programa de CONICYT Programa de Formación de Capital Humano Avanzado Becas de Doctorado en el Extranjero Becas Chile 2018 72190338

\section{REFERENCIAS BIBLIOGRÁFICAS}

1. Institute of Medicine. America's health in transition: protecting and improving quality. Washington (DC): National Academies Press (US); 1994.

2. Donabedian A. Evaluating the quality of medical care. Milbank Mem Fund Q. 1966 Jul;44(3):Suppl:166-206.

3. Donabedian A. The seven pillars of quality. Arch Pathol Lab Med. 1990 Nov;114(11): 1115-8.

4. Ministerio de Salud. Gobierno de Chile. Satisfacción población usuaria hospitales [en línea]. Santiago, Chile; 2012 [consultado el 06 de jun de 2018]. Disponible en: http://www.supersalud.gob.cl/observatorio/671/articles-7596_p5.pdf

5. Arteaga O, Fuentes-García A, Vera A. Anexo estudio nacional de evaluación de percepción del trato a usuarios en establecimientos de salud municipal del sistema público de salud. Santiago, Chile: 2014.

6. Superintendencia de Salud. Estudio de opinión a usuarios del sistema de salud y posicionamiento de la superintendencia de salud. 2016.

7. Unidad de Gestión y Desarrollo, Complejo Asistencial Dr. Sótero del Río. Informe de resultado evaluación de la percepción usuaria en relación al respeto de sus derechos como pacientes enero a diciembre 2017. 2017.
8. Homer CJ, Marino B, Cleary PD, Alpert HR, Smith B, Crowley Ganser CM, et al. Quality of care at a children's hospital: The parents' perspective. Arch Pediatr Adolesc Med. 1999;153(11):1123-9.

9. Zhang C, Report S, Care A, Murray MA. Hospital e-Scorecard Report 2008: Acute Care. Pediatric Patient Satisfaction Technical Summary. 2008.

10. Eulmesekian P. Satisfacción de los padres de los pacientes en una unidad de cuidados intensivos pediátricos. Arch Argent Pediatr. 2012;110(2):113-9.

11. Pautassi LC, Rico MN. Licencias para el cuidado infantil. Derecho de hijos, padres y madres. Boletín Infanc y Adolesc sobre Avance de los Objet Desarro del Milen. 2011;12:4-9.

12. Paul $\mathrm{P}$, Hakobyan $M$, Valtonen $H$. The association between self-perceived health status and satisfaction with healthcare services: Evidence from Armenia. BMC Health Serv Res. 2016;16:67.

13. Xiao H, Barber JP. The effect of perceived health status on patient satisfaction. Value Heal. 2008;11(4):719 25.

14. Schwei RJ, Johnson TP, Matthews AK, Jacobs EA. Perceptions of negative health-care experiences and self-reported health behavior change in three racial and ethnic groups. Ethn Health. 2017;22(2):156-68.

15. Solheim E, Garratt AM. Parent experiences of inpatient pediatric care in relation to health care delivery and sociodemographic characteristics: results of a Norwegian national survey. BMC Health Serv Res. 2013;13:512.

16. Rahmqvist M. Patient satisfaction in relation to age, health status and other background factors: a model for comparisons of care units. Int J Qual Heal care. 2001;13(5):385-90.

17. Rahmqvist M, Bara A-C. Patient characteristics and quality dimensions related to patient satisfaction. Int J Qual Heal Care. 2010;22(2):86-92.

18. Moreno-Monsiváis M, Interial-Guzmán M, Sauceda-Flores P, Vázquez-Arreola L, López-España J. Satisfacción de las madres con la atención a sus hijos hospitalizados. Aquichan. 2011;11(1):40-7.

19. Franck LS, Wray J, Gay C, Dearmun AK, Lee K, Cooper BA. Predictors of parent post-traumatic stress symptoms after child hospitalization on general pediatric wards: A prospective cohort study. Int J Nurs Stud. 2015;52(1):10-21. 
20. Stremler R, Haddad S, Pullenayegum E, Parshuram C. Psychological Outcomes in Parents of Critically III Hospitalized Children. J Pediatr Nurs. 2017;34:36-43.

21. Sigurdardottir AO, Garwick AW, Svavarsdottir EK. The importance of family support in pediatrics and its impact on healthcare satisfaction. Scand J Caring Sci; 2017;31(2):241-52.

22. Lindly OJ, Geldhof GJ, Acock AC, Sakuma K-LK, Zuckerman KE, Thorburn S. Family-Centered Care Measurement and Associations With Unmet Health Care Need Among US Children. Acad Pediatr. 2017;17(6):656-64.

23. Padilla Fortunatti CF. Most Important Needs of Family Members of Critical Patients in Light of the Critical Care Family Needs Inventory. Investig y Educ en Enfermer. 2014;32(2):306-16.

24. Rapport F, Hibbert P, Baysari M, Long JC, Seah R, Zheng $W Y$, et al. What do patients really want? An in-depth examination of patient experience in four Australian hospitals. BMC Health Serv Res. 2019;19(1):38.

25. Batbaatar E, Dorjdagva J, Luvsannyam A, Savino MM, Amenta P. Determinants of patient satisfaction: A systematic review. Perspect Public Health. 2017;137(2):89_ 101.

26. Pinder RJ, Ferguson J, Møller H. Minority ethnicity patient satisfaction and experience: results of the National Cancer Patient Experience Survey in England. BMJ Open. 2016;6(6):e011938.

27. Abuqamar M, Arabiat DH, Holmes S. Parents' Perceived Satisfaction of Care, Communication and Environ- ment of the Pediatric Intensive Care Units at a Tertiary Children's Hospital. J Pediatr Nurs. 2016;31(3):e17784.

28. Eymin G, Aizman A, Lopetegui M, Manjarrez E. Proceso de alta hospitalaria, revisión de la literatura. Rev Med Chil. 2014;142(2):229-37.

29. October TW, Hinds PS, Wang J, Dizon ZB, Cheng YI, Roter DL. Parent Satisfaction With Communication Is Associated With Physician's Patient-Centered Communication Patterns During Family Conferences. Pediatr Crit Care Med. 2016;17(6):490-7.

30. Hagen IH, Iversen VC, Svindseth MF. Differences and similarities between mothers and fathers of premature children: a qualitative study of parents' coping experiences in a neonatal intensive care unit. BMC Pediatr.2016;16:92.

31. Ilioudi S, Lazakidou A, Tsironi M. Importance of Patient Satisfaction Measurement and Electronic Surveys: Methodology and Potential Benefits. Int J Heal Res Innov. $2013 ; 1(1): 67-87$

32. Meesala A, Paul J. Service quality, consumer satisfaction and loyalty in hospitals: Thinking for the future. J Retail Consum Serv. 2018;40:261-9.

33. Institut de la statistique du Québec. Measuring patient experience through surveys : from concepts to best practices. Québec, Canada; 2018.

34. Mehta SJ. Ethics case Patient Satisfaction Reporting and Its Implications for Patient Care Commentary. Am Med Assoc J Ethics. 2015;17(7):616-21. 